\title{
Advances in Theoretical Calculation of Halide Perovskites for Photocatalysis
}

\author{
Xiaolin Liu *, Jichao Fan and Changzhu Huang \\ Department of Physics, Shanghai University of Electric Power, Shanghai, China
}

Photocatalysis, which includes water splitting for hydrogen fuel generation, degradation of organic pollutants, and $\mathrm{CO}_{2}$ reduction using renewable solar energy, is one of the most promising solutions for environmental protection and energy conversion. Halide perovskite has recently emerged as a new promising material for photocatalytic applications. The exploration of new efficient halide perovskite-based photocatalysts and understanding of photocatalytic reaction mechanisms can be revealed using theoretical calculations. The progress and applications of first-principles atomistic modeling and simulation of halide perovskite photocatalysts, including metal halide perovskites, halide perovskite heterojunctions, and other promising perovskite derivatives, are presented in this review. Critical insights into the challenges and future research directions of photocatalysis using halide perovskites are also discussed.

OPEN ACCESS

Edited by:

Yi Yu,

ShanghaiTech University, China

Reviewed by:

Tao Cheng,

Soochow University, China

Zijing Ding,

University of Science and Technology

of China, China

*Correspondence:

Xiaolin Liu

xlliu@shiep.edu.cn

Specialty section: This article was submitted to Nanocatalysis,

a section of the journal

Frontiers in Nanotechnology

Received: 15 April 2021

Accepted: 01 June 2021

Published: 14 June 2021

Citation:

Liu X, Fan J and Huang C (2021) Advances in Theoretical Calculation of Halide Perovskites for Photocatalysis.

Front. Nanotechnol. 3:695490.

doi: 10.3389/fnano.2021.695490
Keywords: first-principles calculations, photocatalysis, halide perovskites, density functional theory, heterojunctions

\section{INTRODUCTION}

As sustainable, inexhaustible, clean, and renewable energy, solar energy has been considered an attractive alternative for applications of heating systems and electricity production in the past decades. Driven by solar energy, photocatalysis is consideredone of the most important routes for using solar energy since the pioneering work on $\mathrm{TiO}_{2}$ for water splitting in 1972 (Fujishima and Honda, 1972). Photocatalytic processes are applied in four fields: water splitting, $\mathrm{CO}_{2}$ reduction, degradation of organic compounds, and organic synthesis. Semiconductors have been the most used photocatalysts because of their intrinsic optical and electronic properties, from conventional binary semiconductor photocatalysts (i.e., g- $\mathrm{C}_{3} \mathrm{~N}_{4}, \mathrm{TiO}_{2}, \mathrm{ZnO}, \mathrm{CdS}$ ) ( $\mathrm{Yu}$ et al., 2013; Jin et al., 2015; Ye et al., 2015; Adhikari et al., 2016; Cheng et al., 2018; Jaramillo-Páez et al., 2018; Yu et al., 2018) to ternary photocatalysts (i.e., $\mathrm{BiVO}_{4}, \mathrm{SrTiO}_{3}, \mathrm{BaTi}_{4} \mathrm{O}_{9}$ ) (Inoue et al., 1992; Kato and Kudo, 2002; Zeng et al., 2018; Andrei et al., 2020). However, conventional photocatalysts have drawbacks, such as large bandgap, high carrier recombination rate, and low light-absorption ability. It is critical to develop new photocatalysts with high photoconversion efficiency.

Metal halide perovskites with a chemical formula of $A B X_{3}\left(\mathrm{~A}=\mathrm{CH}_{3} \mathrm{NH}_{3}, \mathrm{CH}\left(\mathrm{NH}_{2}\right)_{2}, \mathrm{Cs} ; \mathrm{B}=\mathrm{Pb}\right.$, $\mathrm{Sn} ; \mathrm{X}=\mathrm{I}, \mathrm{Br}$, or $\mathrm{Cl}$ ) have recently attracted extensive research attention because of their extraordinary photophysical properties and performance, such as high visible-light absorption coefficients, long charge carrier diffusion lengths, high photoluminescence quantum yield, tunable bandgap, etc (Park et al., 2016; Zhang et al., 2018a; Shi and Jayatissa, 2018). Furthermore, combined with a diversity of A and B sites and ferroelectric and piezoelectric effects (Frost et al., 2014; Kanhere and Chen, 2014; Sun et al., 2016; Ji et al., 2017; Zhu et al., 2019), $A B X_{3}$ halide perovskites are indubitably one of the most promising alternatives in photocatalytic applications (Zhu and Podzorov, 2015; Lee et al., 2018; Hong et al., 2019; Zhu et al., 2019; Chen et al., 2020). 
First-principle atomistic modeling and simulation, such as density functional theory (DFT) and Green's function-based many-body perturbation theory $(G W)$, are fundamental means to investigate material properties by inputting the initial lattice structure and known atomic information. The DFT method is a calculation method based on Hohenberg-Kohn theorem and Kohn-Sham equation (Hohenberg and Kohn, 1964; Kohn and Sham, 1965). When it comes to specific practical problems, it is necessary to consider several choices of exchange-correlation functional, such as Local Density Approximation (LDA), Generalized Gradient Approximation (GGA) and hybrid density functional method (Jones and Gunnarsson, 1989; Parr Robert, 1989).

LDA is mainly aimed at uniform electron gas, meaning that the charge density needs to change slowly in the order of local Fermi wavelength. In the calculation of Raman spectrum and phonon spectrum, LDA tends to a good performance. GGA optimizes the exchange-correlation and adds the gradient of charge density change to the exchange-correlation functional, giving more reasonable simulation results for heterogeneous electronic systems (Richard, 2004; Jiang et al., 2010). The commonly used GGA functionals are Perdew-Burke-Ernzerhof (PBE) (Kresse and Joubert, 1999) and Perdew -Wang (PW91) (Blöchl, 1994). However, GGA ignores the nonlocality of exchange, which usually underestimates the band gap. Therefore, hybrid density functional method is developed, which can improve the calculation accuracy based on the exact exchange energy obtained by Hartree-Fock approximation and the partial exact exchange energy obtained by mixed density functional theory. The commonly used methods are Heyd-Scuseria-Ernzerhof (HSE), PBE0, etc. (Perdew et al., 1996; Heyd et al., 2003) In addition, there are not only correction methods for electronic correlation (Grimme et al., 2010; Cao et al., 2012), such as dispersion interaction correction $(\mathrm{DFT}+D)$, self-interaction correction (DFT $+U$ ), but also correction methods for magnetic materials such as Spin orbit coupling (SOC) (Afsari et al., 2017; Sehgal, 2017).

$G W$ method provides a more rigorous theoretical framework for the theoretical description of the electronic energy band structure of materials, which can give high-precision theoretical predictions for physically simple semiconductor materials, such as $G_{0} W_{0}$ method based on LDA/GGA selfconsistent calculation (Aryasetiawan and Gunnarsson, 1998; Aulbur et al., 2000). However, there is still much room for improvement for physically more complex systems with stronger electronic correlation effects, because LDA/GGA often gives wrong description, which can lead to the wrong calculation of $G_{0} W_{0}$ (Aryasetiawan and Gunnarsson, 1995; Jiang, 2010; Jiang and Zhang, 2020). Many new methods have been developed to choose an appropriate starting point to calculate the reference Hamiltonian, such as $G_{0} W_{0}$ based on DFT + U or HSE (Miyake et al., 2006; Jiang et al., 2010), or quasi particle self-consistent $G W$, and fully self-consistent $G W$ (van Schilfgaarde et al., 2006; Grumet et al., 2018). Among the abovementioned methods, GGA-PBE in DFT calculation method is the most commonly used and inexpensive calculation method, because it does not cost so much computational power, while

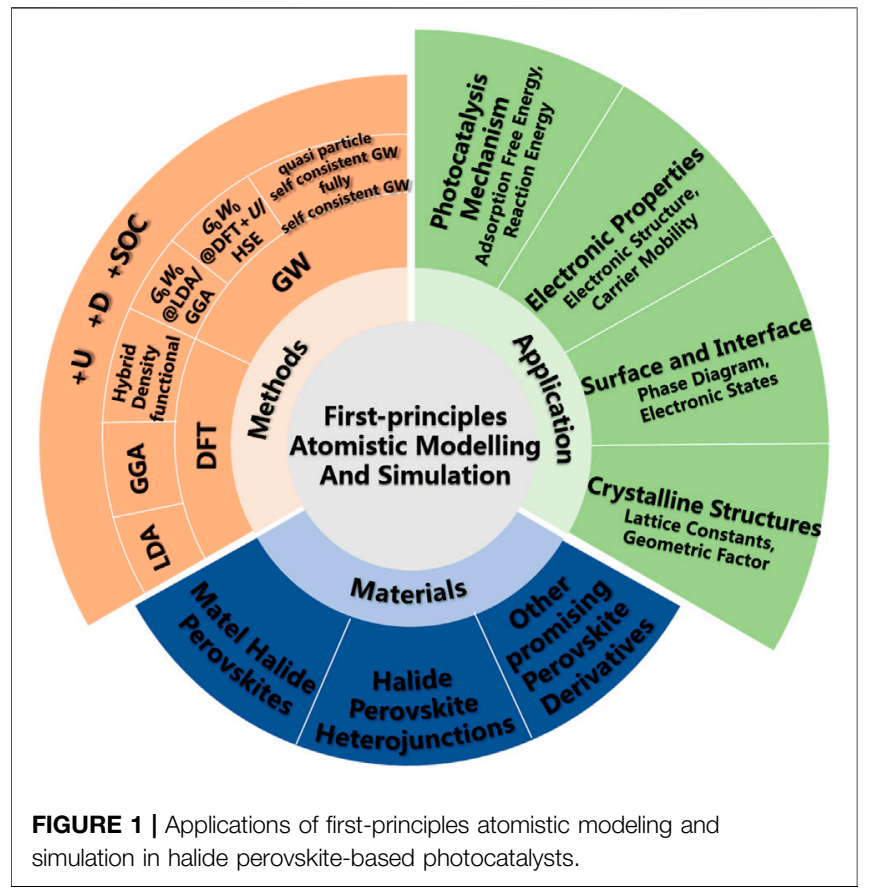

HSE and GW methods are more suitable for dealing with band gap calculation, and there are some problems such as complex program implementation and large amount of calculation.

First-principle methods can be used to explore the lattice constants, electronic structures, carrier mobility and diffusion lengths of photocatalyst, understand the reaction mechanism, and further predict new halide perovskite-based photocatalyst (Catlow et al., 2010; Luo and Daoud, 2015; Yu, 2019). The calculation of these properties is critical for optimizing the photocatalytic performance. Thus far, there has been limited research regarding the booming use of first-principles calculations to study halide perovskites as photocatalysts. Herein, we summarize the recent progress in the theoretical calculation study on halide perovskite-based photocatalysts (Figure 1), and focus on the link between calculated material properties and performance of photocatalysts, including 1) metal halide perovskites, 2) halide perovskite heterojunctions, and 3) other promising perovskite derivatives. Then, we discuss the challenges and future research directions of halide perovskite photocatalysis.

\section{THEORETICAL CALCULATION OF PROPERTIES OF HALIDE PEROVSKITE-BASED PHOTOCATALYSTS Metal Halide Perovskites}

As is well known, for the $A B X_{3}$ perovskites, the $\mathrm{BX}_{6}$ octahedra can be tilted and distorted at finite temperature or pressure (Glazer, 1972), and anharmonic effects are associated with the perovskitephase transition (Buckeridge et al., 2013). Moreover, the bandgap of $A B X_{3}$ perovskites can be tuned by chemical substitution (Sutton et al., 2016; Wang et al., 2019a). Several first- 

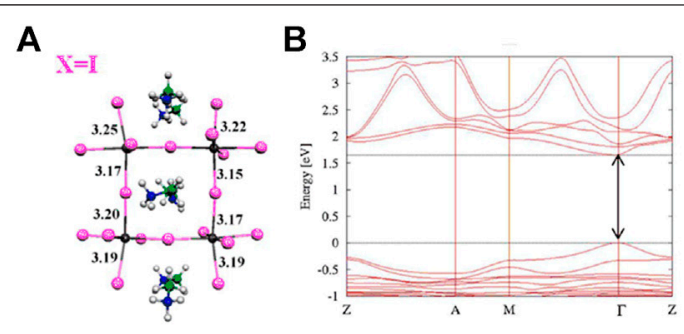

C

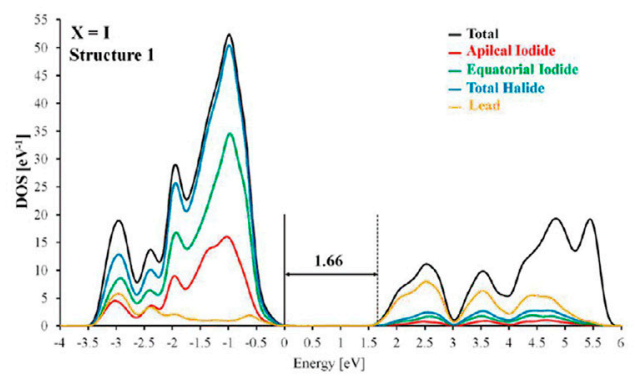

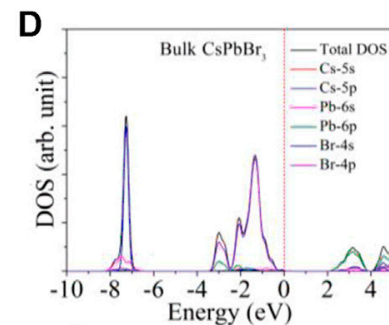
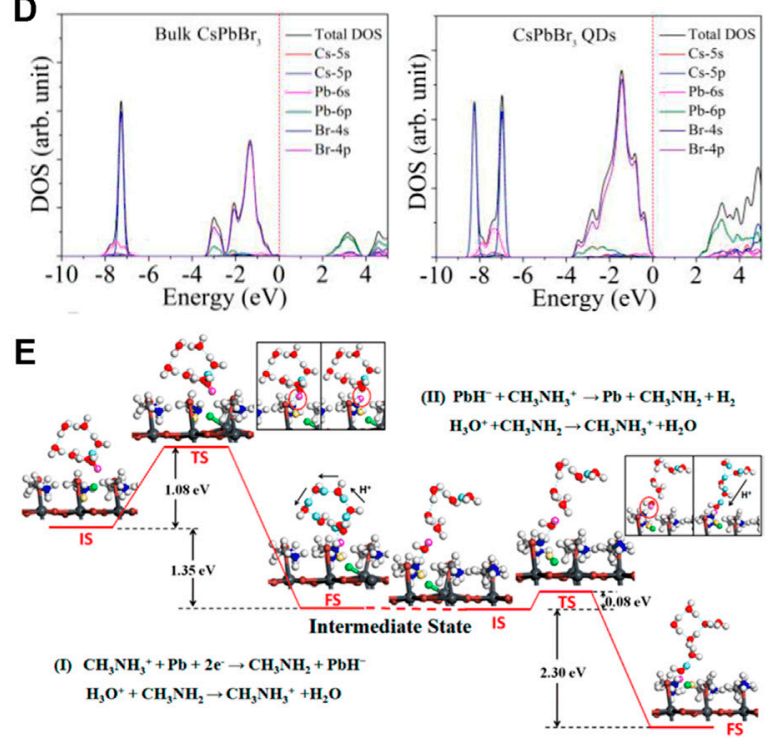

FIGURE 2 | (A) Optimized geometrical structures, (B) calculated band structure, and (C) DOS of MAPbl 3 (Grimme et al., 2010). Copyright (2013) American Chemical Society. (D) Calculated densities of states diagrams of bulk $\mathrm{CsPbBr}_{3}$ and $\mathrm{CsPbBr}_{3}$ QDs (Aryasetiawan and Gunnarsson, 1995). Copyright (2017) John Wiley and Sons. (E) PbAAA reaction pathway for $\mathrm{H}_{2}$ generation on $\mathrm{MAPbl}_{3}$ surface in acidic solvent (Jiang and Zhang, 2020). Copyright (2018) American Chemical Society.

principles investigations into $A B X_{3}$ structures have been conducted to explore their crystal structures, material stability properties, and defect physics. Most notably, the electronic and optical properties of $A B X_{3}$ perovskite as light absorbers in photocatalysis are critical, and several theoretical calculation studies have focused on the electronic energy-band structure, corresponding density of states (DOS), carrier mobility, and other electronic properties of halide perovskite-based photocatalyst.

One of the critical properties of a photocatalyst is its bandgap. Considering the utilization of visible light in the sunlight, the bandgap of $\sim 2 \mathrm{eV}$ is desirable for photocatalytic reaction (Xiaobo et al., 2010). In addition, for $\mathrm{CO}_{2}$ reduction, the position of conduction band minimum (CBM) should be lower than $-0.52 \mathrm{eV}$ (vs. NHE $\mathrm{PH}=0$ ) (the energy barriers of reducing $\mathrm{CO}_{2}$ to CO) (Kanhere and Chen, 2014; Schneider et al., 2012), that is more advantageous to the reduction reaction in thermodynamics. Angelis et al. first computationally investigated the $\mathrm{MAPbX}_{3}$ hybrid halide perovskites in 2013 (Mosconi et al., 2013). They performed periodic DFT calculations within the GGA and found that the calculated bandgap values $\left(\mathrm{MAPbI}_{3}: \mathrm{E}_{g}=1.57 \mathrm{eV}, \mathrm{MAPbBr}_{3}: \mathrm{E}_{g}=\right.$ $1.80 \mathrm{eV}, \mathrm{MAPbCl}_{3}: \mathrm{E}_{g}=2.34 \mathrm{eV}$ ) agreed well with the experimental values. Figures 2A-C shows the optimized geometrical structures, calculated band structure, and DOS of $\mathrm{MAPbI}_{3}$. Afterward, Umari developed an effective GW method incorporating SOC to accurately model the electronic properties of $\mathrm{MAPbI}_{3}$ and $\mathrm{MASnI}_{3}$ (Umari et al., 2014). Furthermore, the HSE06 and PBE functionals, with and without SOC calculations, were used to compute the bandgap of $\mathrm{ABX}_{3}$ halide perovskites (Giorgi et al., 2013; Brivio et al., 2014; Melissen et al., 2015). By comparing the calculated values, it is proved that the strong electron-hole interaction should consider, and the SOC plays a critical role in dominating the conduction band, although the computational requirements increase.

Moreover, the DOS can also be used to predict the performance of photocatalysts through the analysis of total and atomic-resolved DOS. The DOS can be obtained from a non-self-consistent-field (SCF) energy-band calculation, and from the analysis of the partial DOS, the contribution of each site to the valence band and conduction band can be revealed. For $\mathrm{ABX}_{3}$ halide perovskites, the CBM is usually dominated by $\mathrm{B}$ $p$-states, whereas the valence band maximum (VBM) consists of antibonding between $\mathrm{B} s$-states and $\mathrm{X} p$-states (Borriello et al., 2008; Chung et al., 2012; Mosconi et al., 2013; Umari et al., 2014). Furthermore, the photogenerated carrier transfer ability can be revealed by the calculated DOS. Sun et al. synthesized colloidal $\mathrm{CsPBBr}_{3}$ quantum dots (QDs) as new photocatalytic material (Hou et al., 2017). To understand the advantages of the quantum size effect, they calculated the DOS of bulk $\mathrm{CsPbBr}_{3}$ and atomic $\mathrm{CsPbBr}_{3}$ QD slabs. Different from the bulk $\mathrm{CsPbBr}$, the conduction band of $\mathrm{CsPbBr}_{3}$ QDs was primarily contributed by the coupling of $\mathrm{Cs} p$-states, $\mathrm{Br} p$-states, and $\mathrm{Pb} p$-states, whereas the valence band was constructed by the coupling of $\mathrm{Cs} p$-states, $\mathrm{Pb} s$-states, and $\mathrm{Br} p$-states (as shown in Figure 2D). Due to the increased DOS at CBM, more photogenerated carriers can reach $\mathrm{CBM}$, resulting in more efficient photochemical conversion.

The last, but the most important issue is the photocatalytic mechanism. Metal halide perovskites have been successfully confirmed as efficient photocatalysts. However, it is difficult to develop improved perovskite materials without a thorough analysis of reaction mechanisms. Wang et al. derived the photocatalytic hydrogen evolution reaction (HER) mechanism using PBE functional of DFT, including the D3 van der Waals 

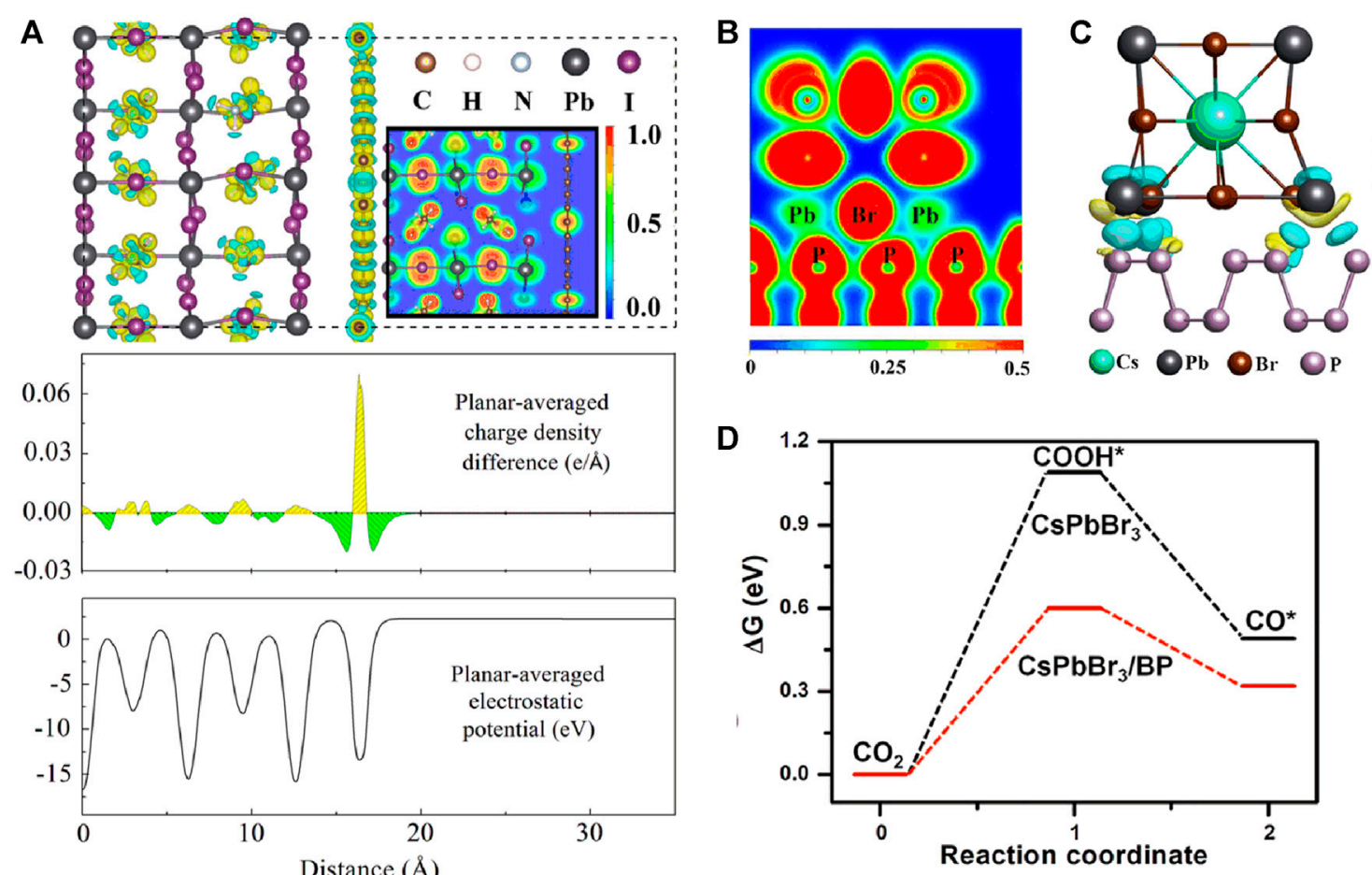

FIGURE 3 | (A) Calculated electrostatic potential, charge density difference, and electron localization function plots for graphdiyne/MAPbl 3 heterostructures with five-layer interfaces (Glazer, 1972). Copyright (2019) John Wiley and Sons. (B) Electron localization function, and (C) charge density difference of the interface between the $\mathrm{CsPbBr}_{3}$ and black phosphorus (Yu, 2019). (D) Gibbs free-energy diagram of $\mathrm{CsPbBr}_{3} / \mathrm{black}$ phosphorus and $\mathrm{CsPbBr}_{3}$ heterostructures in the photocatalytic $\mathrm{CO}_{2}$ reduction process (Yu, 2019). Copyright (2020) Elsevier.

correction to account for London dispersion interactions (Zhang et al., 2018b). They constructed a supercell of the (010) surface of $\mathrm{MAPbI}_{3}$ in $\mathrm{HI}$ aqueous solution, and by comparing the activation barriers and reaction energies of different reaction pathways, they found that the $\mathrm{Pb}$ atoms and surface organic molecules play critical roles in the HER reaction. Furthermore, a two-step Pbactivated amine-assisted (PbAAA) reaction mechanism was proposed (Figure 2E), which is widely accepted and approved. Tao et al. performed the DFT calculations with the PbAAA mechanism to explain the $\mathrm{Br}$ effect on the enhanced performance of the $\mathrm{MAPb}\left(\mathrm{I}_{1-\mathrm{x}} \mathrm{Br}_{\mathrm{x}}\right)_{3}$ photocatalytic HER process (Zhao et al., 2019a). Due to the smaller $\mathrm{Br}$ ionic radius, the $\mathrm{Br}-\mathrm{Pb}$ bonds can be easily broken, and more $\mathrm{Pb}$ defect sites were formed for lowering the $\mathrm{H}-\mathrm{Pb}$ absorption energy, and thus $\mathrm{MAPb}\left(\mathrm{I}_{1-\mathrm{x}} \mathrm{Br}_{\mathrm{x}}\right)_{3}$ shows a superb HER rate under visible light illumination.

\section{Halide Perovskite Heterojunctions}

To enhance the photocatalytic performance, the construction of halide perovskite heterojunctions with two-dimensional (2D) materials such as graphene, $\mathrm{g}-\mathrm{C}_{3} \mathrm{~N}_{4}$, and black phosphorus has caused extensive concern due to improved stability, more efficient carrier mobility, and regulated bandwidth (Xu et al., 2017; Ou et al., 2018; Wu et al., 2018; Zhao et al., 2019b; Guo et al., 2019; Paul et al., 2019; Wang, 2020). For the heterojunction calculations, the main problem is the understanding of the charge density difference and charge transfer process at the contacting interface.

Guo et al. used density-functional first-principles calculations with empirical dispersion corrections to investigate the interfacial interactions and electronic properties of graphdiyne/MAPbI heterostructures (Guo et al., 2019). By employing the projector-augmented wave (PAW) to describe the electroncore interaction, and GGA of PBE as the exchange-correlation functional, they found that the graphdiyne/ $\mathrm{MAPbI}_{3}$ heterostructure was formed by van der Waals interaction, and the $\mathrm{PbI}_{2}$-terminated surface showed high stability when contacting graphdiyne. Combining HSE06 with SOC, the electronic properties including charge density difference, electron localization field, Bader charge, and electrostatic potential were analyzed, as shown in Figure 3A. The electronhole separation is obviously enhanced due to the built-in electric field at the interface.

In addition to the van der Waals interaction of the heterostructure interface, $\mathrm{Zhu}$ et al. found that a significant covalent bond between $\mathrm{CsPbBr}_{3}$ and black phosphorus nanosheets was formed in the heterostructure by DFT calculation (Wang, 2020). Moreover, charge density difference analysis revealed that a directional electron transfer channel was generated reasonably between $\mathrm{Pb}$ and $p$ elements, as shown in Figure 3B,C which was consistent with the experimental results. Furthermore, using $a b$ initio atomistic thermodynamics 


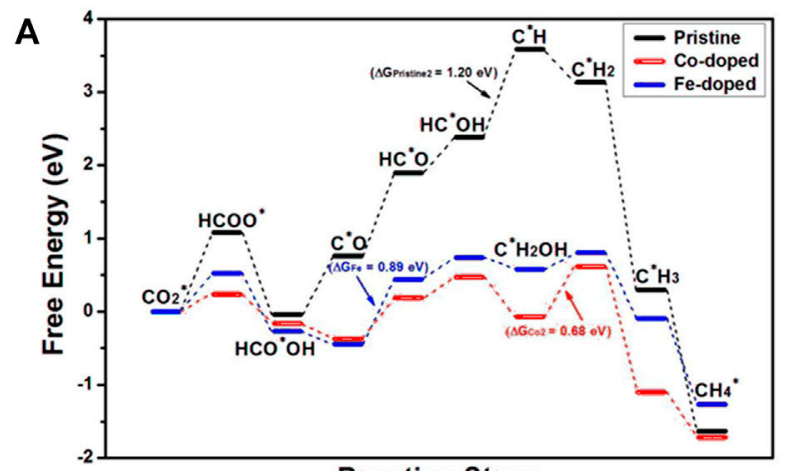

Reaction Steps

B
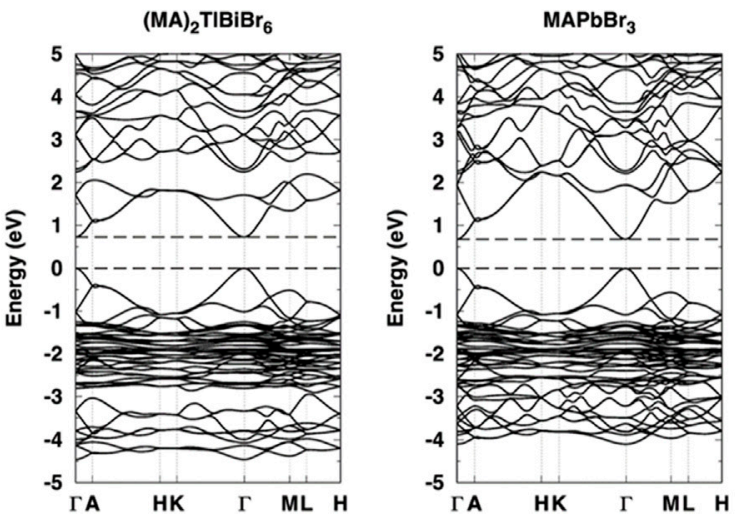

C

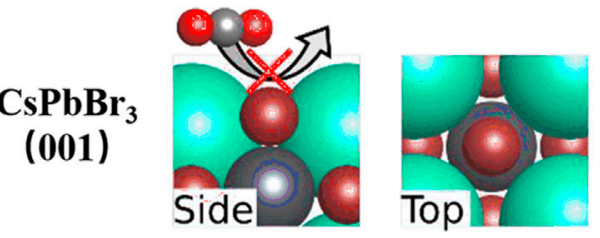

D
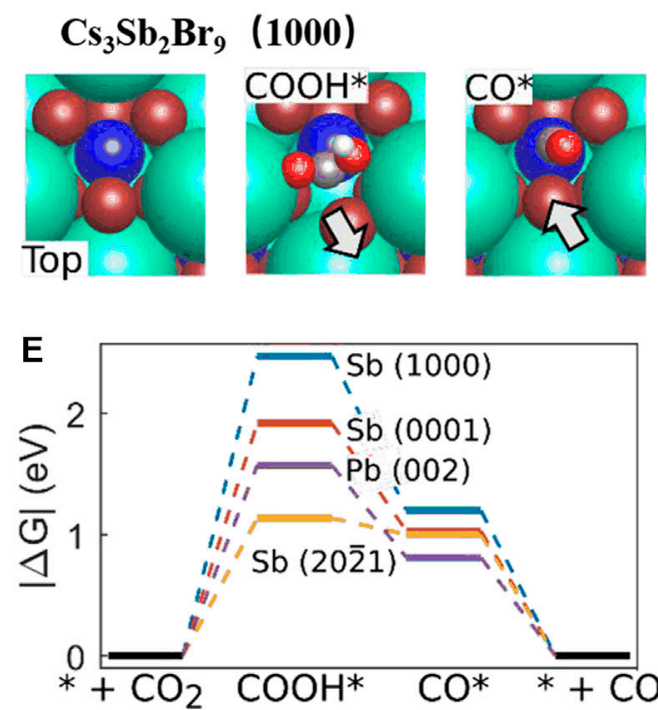

FIGURE 4 | (A) The free-energy diagrams of the most favored paths of $\mathrm{CO}_{2}$ reduction on pristine, Co-doped and Fe-doped CsPbBr 3 (Umari et al., 2014). Copyright (2019) Royal Society of Chemistry. (B) Calculated electronic band structures of (MA) ${ }_{2} \mathrm{TIBiBr}_{6}$ and $\mathrm{MAPbBr}_{3}$ (Melissen et al., 2015). Copyright (2016) Royal Society of Chemistry. Schematic of the (C) $\mathrm{CsPbBr}_{3}$ (001) surface and (D) reactivity of highly exposed $\mathrm{Cs}_{3} \mathrm{Sb}_{2} \mathrm{Br}_{9}$ (1000) surface. (E) Free-energy pathways for highly exposed $\mathrm{Cs}_{3} \mathrm{Sb}_{2} \mathrm{Br}_{9}$ (1000), (0001) and (2021) surfaces and $\mathrm{CsPbBr}_{3}$ (002) surface (Giorgi et al., 2013). Copyright (2020) Royal Society of Chemistry.

calculations, the possible mechanism of $\mathrm{CO}_{2}$ reduction by $\mathrm{CsPbBr}_{3}$ and black phosphorus nanosheet heterostructure was clarified. The black phosphorus nanosheets acted as the electron acceptor, which promoted the breaking of the $\mathrm{C}=\mathrm{O}$ bonds and reduced the reaction barrier energy (Figure 3D).

\section{Other Promising Perovskite Derivatives}

The exploration of new photocatalysts is an important way to achieve high photocatalytic performance. And the new promising halide perovskites offer distinct advantages over the traditional perovskite materials (Volonakis et al., 2016; Liu et al., 2018; Maughan et al., 2018; Yan et al., 2018; Tang et al., 2019; Yue et al., 2020), such as substitution or mixing, double perovskites, and other perovskite derivatives. Theoretical calculation is a powerful tool to predict new functional perovskite materials suitable for photocatalysis and optimize the stoichiometric ratio of mixed halide perovskites. Especially when introducing the Goldschmidt tolerance factor $t=\left(r_{A}+r_{X}\right) /\left[\sqrt{2}\left(r_{B}+r_{X}\right)\right]$ (where $r_{A}, r_{B}$ and $r_{X}$ refer to ionic radii for ions in $A, B$ and $X$ sites) (Goldschmidt, 1926), a useful parameter for basic formability prediction of any new materials with targeting perovskite structure, into the first-principle calculations, many studies have selected several promising perovskite materials from hundreds of compounds (Bartel et al., 2020; Fedorovskiy et al., 2020).

$\mathrm{Xu}$ et al. compared the catalytic efficiency of bulk $\mathrm{CsPbBr}$ doped with different metals (Co., Fe, $\mathrm{Ni}, \mathrm{Cu}, \mathrm{Ag}, \mathrm{Mg}, \mathrm{Mn}$, and $\mathrm{Bi}$ ) and concluded that Co. and Fe doping could be ideal for $\mathrm{CO}_{2}$ reduction (Tang et al., 2019). They specifically analyzed the adsorption-free energy using GGA of PBE functional with the PAW method. As shown in Figure 4A the ab initio molecular dynamics calculations indicated that $\mathrm{Co}-$ and $\mathrm{Fe}$-doped systems showed stronger $\mathrm{CO}_{2}{ }^{*}$ adsorption energy (* refers to the adsorption site), which is the most important factor to determine the catalytic efficiency. Furthermore, using HSE06 with DFT + U correction to calculate the Hirshfeld charges and DOS of the strongly correlated effect of Co. atoms, they further illustrated the proposed reaction mechanism and confirmed that $\mathrm{Co}$-doped $\mathrm{CsPBr}_{3}$ could be a great candidate for $\mathrm{CO}_{2}$ reduction.

The toxicity of lead limits the wide commercialization of metal halide perovskite, and thus lead-free alternatives have recently attracted great attention, such as double perovskites $\left(A_{2} B X_{6}\right.$ or $\left.A_{2} B^{+1} B^{+3} X_{6}\right)$ and other perovskite derivatives $\left(A_{3} B_{2} X_{9}, A_{3} B X_{5}\right.$, $A_{4} B X_{6}$, etc.), where $\mathrm{Pb}^{2+}$ cations are replaced using other metal cations, e.g., $\mathrm{Na}^{+}, \mathrm{Ag}^{+}, \mathrm{Bi}^{3+}$, and $\mathrm{Sb}^{3+}$. It is relatively difficult to experimentally synthesize thin films of these materials for 
photovoltaic and photocatalysis applications. However, the theoretical calculation is extremely useful because of its predicting power. For example, Cheetham et al. have used DFT calculations to compare the bandgaps and electronic structures of $(\mathrm{MA})_{2} B^{+1} \mathrm{BiX}_{6}\left(\mathrm{~B}^{+1}=\right.$ e.g., $\left.\mathrm{K}^{+1}, \mathrm{Ag}^{+1}, \mathrm{Cu}^{+1}, \mathrm{Tl}^{+1}\right)$ and $\mathrm{MAPbX}_{3}$, and found that the new double perovskite $(\mathrm{MA})_{2} \mathrm{TL}^{+1} \mathrm{BiBr}_{6}$, had strikingly isoelectronic properties with $\mathrm{MAPbBr}_{3}$, as shown in Figure 4 2) (Deng et al., 2016). This study provided an intriguing alternative to the lead-containing $\mathrm{MAPb} X_{3}$ for photoelectronic applications.

For the all-inorganic halide double perovskites, Giustino et al. (Volonakis et al., 2017) proposed a new promising halide double perovskites, $\mathrm{Cs}_{2} \operatorname{In} \mathrm{Ag} X_{6}$, with the direct band gaps between the visible and the ultraviolet as computed by the HSE06 and PBE0. Recently, Bartel et al. (Bartel et al., 2020) predicted 47 likely synthesizable compounds containing non toxic elements and with direct or nearly direct band gaps among the 311 cesium chloride double perovskites $\left(\mathrm{Cs}_{2} \mathrm{BB}^{\prime} \mathrm{Cl}_{6}\right)$ compounds by computing with HSE06, and identified that the triple-alkali perovskites (where $B$ refers to a group 1 alkali, and $B^{\prime}$ refers to a transition-metal cation) showed remarkable optical properties as computed by the $G W$-Bethe-Salpeter equation method. These studies are helpful to realize the future studies aim and synthesize more efficient photocatalyst with unique optoelectronic properties.

Besides the analyses of the structural, electronic and optical properties, first-principles calculations can also be used to predict phase transition temperatures, which have instructive significance on the exploration of new materials. Kye et al. calculated the bandgaps and optical properties of $\mathrm{K}_{2} \mathrm{Sn} X_{6}(\mathrm{X}=$ $\mathrm{I}, \mathrm{Br}, \mathrm{Cl}$ ) in cubic, tetragonal, and monoclinic phases, and concluded that the cubic $\mathrm{K}_{2} \mathrm{SnBr}_{6}$ and monoclinic $\mathrm{K}_{2} \mathrm{SnI}_{6}$ can be promising candidates for light absorber materials (Jong et al., 2020). Moreover, they calculated the Helmholtz-free-energy differences among the above three phases, and gave the phase transition temperatures of $\mathrm{K}_{2} \mathrm{Sn} X_{6}$, respectively.

As mentioned above, it is difficult to experimentally synthesize uniform thin films of double perovskites or other perovskite derivatives; however, nanocrystals or other nanostructures of these materials can be synthesized more easily. Furthermore, they are proved to be promising candidates because of their highly exposed surface. Geyer et al. developed $\mathrm{Cs}_{3} \mathrm{Sb}_{2} \mathrm{Br}_{9}$ nanocrystals with significantly improved photocatalytic $\mathrm{CO}$ production efficiency ( $\mathrm{Lu}$ et al., 2020). Moreover, the analogous mechanism was observed using DFT calculations, as shown in Figures 4C-E. They calculated the surface energies and adsorption free energies of the $(0,001)(1,000)$, and $(20 \overline{2} 1)$ surfaces of $\mathrm{Cs}_{3} \mathrm{Sb}_{2} \mathrm{Br}_{9}$ as well as the (001) and (002) surfaces of $\mathrm{CsPbrr}_{3}$, and found that the surfaces of $\mathrm{Cs}_{3} \mathrm{Sb}_{2} \mathrm{Br}_{9}$ had a lower free-energy for the adsorption of $\mathrm{COOH}^{\star}$ and $\mathrm{CO}^{\star}$ intermediates because of the predominant defects, which suggested the enhanced catalysis activity.

As mentioned above, perovskite heterojunctions are usually endowed with optimized charge carrier dynamics for improved photocatalytic performance, and perovskite derivative heterojunctions are no exception (Feng et al., 2017; Guo et al., 2017; Wang et al., 2019b). By employing the DFT calculation,
Kuang et al. (Wang et al., 2019b) investigated the electronic structures and interfacial properties in the $\mathrm{Cs}_{2} \mathrm{SnI}_{6} / \mathrm{SnS}_{2}$ hybrid heterojunctions. Then, they revealed that a typical type II band alignment formed in the heterojunction, which was in accordance with the experimental values. Due to the facilitated charge separation between $\mathrm{Cs}_{2} \mathrm{SnI}_{6}$ and $\mathrm{SnS}_{2}$ (i.e, holes from $\mathrm{SnS}_{2}$ to $\mathrm{Cs}_{2} \mathrm{SnI}_{6}$ and electrons from $\mathrm{Cs}_{2} \mathrm{SnI}_{6}$ to $\mathrm{SnS}_{2}$ ), the $\mathrm{CO}_{2}$ photocatalytic reduction activity was unquestionably boosted.

We believed that the theoretical calculation of perovskite derivatives and heterojunction can help predict more direct or nearly direct band-gap materials and choose some more matching materials forming heterojunction with promoted the charge carrier mobility and reduced charge carrier recombination.

\section{CONCLUSION AND OUTLOOK}

Herein, we summarized current computational work on the halide perovskite-based photocatalyst. To reveal the underlying photocatalytic mechanisms, first-principles calculations were used to investigate the crystal structures, electronic properties, defect physics, and surface and interface interaction of perovskites. Furthermore, the promising potential of the firstprinciples material design for predicting new halide perovskites such as substitution or mixing, double perovskites, or other perovskite derivatives has also been demonstrated.

From our perspective, several critical issues and challenges still need to be overcome. First, several unique properties of halide perovskites, such as piezoelectric, and ferroelectric properties due to the anharmonicity in lattice dynamics should be illustrated and explored to promote the photocatalytic reaction efficiency. Second, the influence of structure on the photocatalytic performance of halide perovskite-based heterojunctions, such as the types of ligands, defects, dopants, and crystalline phases, needs to be further researched to improve the activity of the heterostructures. Third, theoretical analytical models of perovskite derivatives can be helpful to promote the charge carrier mobility and reduce charge carrier recombination.

\section{AUTHOR CONTRIBUTIONS}

XL proposed the research idea and manuscript; JF and $\mathrm{CH}$ performed check collection, collation and analysis; All authors reviewed the manuscript, have given approval to the final version of the manuscript, and confirmed the authorship to this work.

\section{FUNDING}

This work was supported by the National Natural Science Foundation of China (Grant No. 61875119), Shanghai Rising-Star Program (Grant No. 19QA1404000), and the "Chen Guang" project supported by Shanghai Municipal Education Commission and Shanghai Education Development Foundation (Grant No. 18CG63). 


\section{REFERENCES}

Adhikari, S. P., Hood, Z. D., More, K. L., Chen, V. W., and Lachgar, A. (2016). A Visible-Light-Active Heterojunction with Enhanced Photocatalytic Hydrogen Generation. ChemSusChem 9 (14), 1869-1879. doi:10.1002/cssc.201600424

Afsari, M., Boochani, A., Hantezadeh, M., and Elahi, S. M. (2017). Topological Nature in Cubic Phase of Perovskite CsPbI 3 : By DFT. Solid State. Commun. 259, 10-15. doi:10.1016/j.ssc.2017.04.014

Andrei, V., Reuillard, B., and Reisner, E. (2020). Bias-free Solar Syngas Production by Integrating a Molecular Cobalt Catalyst with Perovskite-BiVO4 Tandems. Nat. Mater. 19 (2), 189-194. doi:10.1038/s41563-019-0501-6

Aryasetiawan, F., and Gunnarsson, O. (1995). Electronic Structure of $\mathrm{NiO}$ in theGWApproximation. Phys. Rev. Lett. 74 (16), 3221-3224. doi:10.1103/ physrevlett.74.3221

Aryasetiawan, F., and Gunnarsson, O. (1998). TheGWmethod. Rep. Prog. Phys. 61 (3), 237-312. doi:10.1088/0034-4885/61/3/002

Aulbur, W. G., Jönsson, L., and Wilkins, J. W. (2000). "Quasiparticle Calculations in Solids," in Solid State Physics. Editors H. Ehrenreich and F. Spaepen (Academic Press), 1-218. doi:10.1016/s0081-1947(08)60248-9

Bartel, C. J., Clary, J. M., Sutton, C., Vigil-Fowler, D., Goldsmith, B. R., Holder, A. M., et al. (2020). Inorganic Halide Double Perovskites with Optoelectronic Properties Modulated by Sublattice Mixing. J. Am. Chem. Soc. 142 (11), 5135-5145. doi:10.1021/jacs.9b12440

Blöchl, P. E. (1994). Projector Augmented-Wave Method. Phys. Rev. B 50 (24), 17953-17979. doi:10.1103/physrevb.50.17953

Borriello, I., Cantele, G., and Ninno, D. (2008). Ab Initio investigation of Hybrid Organic-Inorganic Perovskites Based on Tin Halides. Phys. Rev. B 77 (23), 235214. doi:10.1103/physrevb.77.235214

Brivio, F., Butler, K. T., Walsh, A., and Schilfgaarde, M. V. (2014). Relativistic Quasiparticle Self-Consistent Electronic Structure of Hybrid Halide Perovskite Photovoltaic Absorbers. Phys. Rev. B 89 (15), 155204. doi:10.1103/physrevb.89. 155204

Buckeridge, J., Scanlon, D. O., Walsh, A., Catlow, C. R. A., and Sokol, A. A. (2013). Dynamical Response and Instability in Ceria under Lattice Expansion. Phys. Rev. B 87 (21), 214304. doi:10.1103/physrevb.87.214304

Cao, J., Li, X., Lin, H., Chen, S., and Fu, X. (2012). In Situ preparation of Novel P-N junction Photocatalyst $\mathrm{BiOI} /(\mathrm{BiO}) 2 \mathrm{CO} 3$ with Enhanced Visible Light Photocatalytic Activity. J. Hazard. Mater. 239-240, 316-324. doi:10.1016/j. jhazmat.2012.08.078

Catlow, C. R. A., Guo, Z. X., Miskufova, M., Shevlin, S. A., Smith, A. G. H., Sokol, A. A., et al. (2010). Advances in Computational Studies of Energy Materials. Phil. Trans. R. Soc. A. 368, 3379-3456. doi:10.1098/rsta.2010.0111

Chen, P., Ong, W-J., Shi, Z., Zhao, X., and Li, N. (2020). Pb-Based Halide Perovskites: Recent Advances in Photo(electro)catalytic Applications and Looking beyond. Adv. Funct. Mater. 30 (30). doi:10.1002/adfm.201909667

Cheng, L., Xiang, Q., Liao, Y., and Zhang, H. (2018). CdS-Based Photocatalysts. Energy Environ. Sci. 11 (6), 1362-1391. doi:10.1039/c7ee03640j

Chung, I., Song, J.-H., Im, J., Androulakis, J., Malliakas, C. D., Li, H., et al. (2012). CsSnI3: Semiconductor or Metal? High Electrical Conductivity and Strong Near-Infrared Photoluminescence from a Single Material. High Hole Mobility and Phase-Transitions. J. Am. Chem. Soc. 134 (20), 8579-8587. doi:10.1021/ ja301539s

Deng, Z., Wei, F., Sun, S., Kieslich, G., Cheetham, A. K., and Bristowe, P. D. (2016). Exploring the Properties of lead-free Hybrid Double Perovskites Using a Combined Computational-Experimental Approach. J. Mater. Chem. A. 4 (31), 12025-12029. doi:10.1039/c6ta05817e

Fedorovskiy, A. E., Drigo, N. A., and Nazeeruddin, M. K. (2020). The Role of Goldschmidt's Tolerance Factor in the Formation of A 2 BX 6 Double Halide Perovskites and its Optimal Range. Small Methods 4 (5), 1900426. doi:10.1002/ smtd.201900426

Feng, H.-J., Deng, W., Yang, K., Huang, J., and Zeng, X. C. (2017). Double Perovskite Cs2BBiX6 $(\mathrm{B}=\mathrm{Ag}, \mathrm{Cu} ; \mathrm{X}=\mathrm{Br}, \mathrm{Cl}) / \mathrm{TiO} 2$ Heterojunction: $\mathrm{An}$ Efficient $\mathrm{Pb}$-free Perovskite Interface for Charge Extraction. J. Phys. Chem. C 121 (8), 4471-4480. doi:10.1021/acs.jpcc.7b00138

Frost, J. M., Butler, K. T., Brivio, F., Hendon, C. H., van Schilfgaarde, M., and Walsh, A. (2014). Atomistic Origins of High-Performance in Hybrid
Halide Perovskite Solar Cells. Nano Lett. 14 (5), 2584-2590. doi:10. $1021 /$ nl500390f

Fujishima, A., and Honda, K. (1972). Electrochemical Photolysis of Water at a Semiconductor Electrode. Nature 238 (5358), 37-38. doi:10.1038/238037a0

Giorgi, G., Fujisawa, J.-I., Segawa, H., and Yamashita, K. (2013). Small Photocarrier Effective Masses Featuring Ambipolar Transport in Methylammonium Lead Iodide Perovskite: A Density Functional Analysis. J. Phys. Chem. Lett. 4 (24), 4213-4216. doi:10.1021/jz4023865

Glazer, A. M. (1972). The Classification of Tilted Octahedra in Perovskites. Acta Crystallogr. Sect B 28 (11), 3384-3392. doi:10.1107/s0567740872007976

Goldschmidt, V. M. (1926). Die Gesetze der Krystallochemie. Naturwissenschaften 14 (21), 477-485. doi:10.1007/bf01507527

Grimme, S., Antony, J., Ehrlich, S., and Krieg, H. (2010). A Consistent and Accurate $\mathrm{Ab}$ Initio Parametrization of Density Functional Dispersion Correction (DFT-D) for the 94 Elements H-Pu. J. Chem. Phys. 132 (15), 154104. doi:10.1063/1.3382344

Grumet, M., Liu, P., Kaltak, M., Klimeš, J., and Kresse, G. (2018). Beyond the Quasiparticle Approximation: Fully Self-Consistent GW Calculations. Phys. Rev. B 98 (15), 155143. doi:10.1103/physrevb.98.155143

Guo, Y., Saidi, W. A., and Wang, Q. (2017). 2D halide perovskite-based van der Waals heterostructures: contact evaluation and performance modulation. $2 D$ Mater. 4 (3), 035009. doi:10.1088/2053-1583/aa7ac3

Guo, Y., Xue, Y., Li, C., and Li, X. (2019). Electronic Properties of the Graphdiyne/ CH3NH3PbI3 Interface: A First-Principles Study. physica status solidi (Rrl) Rapid Res. Lett. 14 (1). doi:10.1002/pssr.201900544

Heyd, J., Scuseria, G. E., and Ernzerhof, M. (2003). Hybrid Functionals Based on a Screened Coulomb Potential. J. Chem. Phys. 118 (18), 8207-8215. doi:10.1063/ 1.1564060

Hohenberg, P., and Kohn, W. (1964). Inhomogeneous Electron Gas. Phys. Rev. 136 (3B), B864-B871. doi:10.1103/physrev.136.b864

Hong, Z., Chong, W. K., Ng, A. Y. R., Li, M., Ganguly, R., Sum, T. C., et al. (2019). Hydrophobic Metal Halide Perovskites for Visible-Light Photoredox C-C Bond Cleavage and Dehydrogenation Catalysis. Angew. Chem. Int. Ed. 58 (11), 3456-3460. doi:10.1002/anie.201812225

Hou, J., Cao, S., Wu, Y., Gao, Z., Liang, F., Sun, Y., et al. (2017). Inorganic Colloidal Perovskite Quantum Dots for Robust Solar CO 2 Reduction. Chem. Eur. J. 23 (40), 9481-9485. doi:10.1002/chem.201702237

Inoue, Y., Niiyama, T., Asai, Y., and Sato, K. (1992). Stable Photocatalytic Activity of BaTi4O9 Combined with Ruthenium Oxide for Decomposition of Water. J. Chem. Soc. Chem. Commun. (7), 579-580. doi:10.1039/c39920000579

Jaramillo-Páez, C. A., Navío, J. A., Hidalgo, M. C., and Macías, M. (2018). ZnO and Pt-ZnO Photocatalysts: Characterization and Photocatalytic Activity Assessing by Means of Three Substrates. Catal. Today 313, 12-19. doi:10.1016/j.cattod. 2017.12.009

Ji, C., Sun, Z., Zeb, A., Liu, S., Zhang, J., Hong, M., et al. (2017). Bandgap Narrowing of Lead-Free Perovskite-type Hybrids for Visible-Light-Absorbing Ferroelectric Semiconductors. J. Phys. Chem. Lett. 8 (9), 2012-2018. doi:10.1021/acs.jpclett. $7 \mathrm{~b} 00673$

Jiang, H., Gomez-Abal, R., Rinke, P., and Scheffler, M. (2010). First-principles Modeling of Localized D States with the GW@LDA+U Approach. Phys. Rev. B 82 (4), 045108. doi:10.1103/physrevb.82.045108

Jiang, H. (2010). The GW Method: Basic Principles, Latest Developments and its Applications for D -and F -Electron Systems. Acta Phys. -Chim. Sin. 26 (04), 1017-1033.

Jiang, H., and Zhang, M. (2020). Density-functional Theory Methods for Electronic Band Structure Properties of Materials. SCIENTIA SINICA Chim. 50 (16747224), 1344

Jin, J., Yu, J., Guo, D., Cui, C., and Ho, W. (2015). A Hierarchical Z-Scheme CdSWO3Photocatalyst with Enhanced CO2Reduction Activity. Small 11 (39), 5262-5271. doi:10.1002/smll.201500926

Jones, R. O., and Gunnarsson, O. (1989). The Density Functional Formalism, its Applications and Prospects. Rev. Mod. Phys. 61 (3), 689-746. doi:10.1103/ revmodphys.61.689

Jong, U.-G., Yu, C.-J., and Kye, Y.-H. (2020). Computational Prediction of Structural, Electronic, and Optical Properties and Phase Stability of Double Perovskites K2SnX6 (X = I, Br, Cl). RSC Adv. 10 (1), 201-209. doi:10.1039/ c9ra09232c 
Kanhere, P., and Chen, Z. (2014). A Review on Visible Light Active PerovskiteBased Photocatalysts. Molecules 19 (12), 19995-20022. doi:10.3390/ molecules191219995

Kato, H., and Kudo, A. (2002). Visible-Light-Response and Photocatalytic Activities of $\mathrm{TiO} 2$ and $\mathrm{SrTiO} 3$ Photocatalysts Codoped with Antimony and Chromium. J. Phys. Chem. B 106 (19), 5029-5034. doi:10.1021/ jp0255482

Kohn, W., and Sham, L. J. (1965). Quantum Density Oscillations in an Inhomogeneous Electron Gas. Phys. Rev. 137 (6A), A1697-A1705. doi:10. 1103/physrev.137.a1697

Kresse, G., and Joubert, D. (1999). From Ultrasoft Pseudopotentials to the Projector Augmented-Wave Method. Phys. Rev. B 59 (3), 1758-1775. doi:10. 1103/physrevb.59.1758

Lee, L. C., Huq, T. N., MacManus-Driscoll, J. L., and Hoye, R. L. Z. (2018). Research Update: Bismuth-Based Perovskite-Inspired Photovoltaic Materials. APL Mater. 6 (8), 084502. doi:10.1063/1.5029484

Liu, Y.-L., Yang, C.-L., Wang, M.-S., Ma, X.-G., and Yi, Y.-G. (2018). Theoretical Insight into the Optoelectronic Properties of lead-free Perovskite Derivatives of Cs3Sb2X9 (X = Cl, Br, I). J. Mater. Sci. 54 (6), 4732-4741. doi:10.1007/s10853018-3162-y

Lu, C., Itanze, D. S., Aragon, A. G., Ma, X., Li, H., Ucer, K. B., et al. (2020). Synthesis of lead-free Cs3Sb2Br9 Perovskite Alternative Nanocrystals with Enhanced Photocatalytic CO2 Reduction Activity. Nanoscale 12 (5), 2987-2991. doi:10. 1039/c9nr07722g

Luo, S., and Daoud, W. A. (2015). Recent Progress in Organic-Inorganic Halide Perovskite Solar Cells: Mechanisms and Material Design. J. Mater. Chem. A. 3 (17), 8992-9010. doi:10.1039/c4ta04953e

Richard, M. M. (2004). Electronic Structure: Basic Theory and Practical Methods. Cambridge: Cambridge University Press.

Maughan, A. E., Ganose, A. M., Almaker, M. A., Scanlon, D. O., and Neilson, J. R. (2018). Tolerance Factor and Cooperative Tilting Effects in Vacancy-Ordered Double Perovskite Halides. Chem. Mater. 30 (11), 3909-3919. doi:10.1021/acs. chemmater.8b01549

Melissen, S. T. A. G., Labat, F., Sautet, P., and Le Bahers, T. (2015). Electronic Properties of $\mathrm{PbX} 3 \mathrm{CH} 3 \mathrm{NH} 3(\mathrm{X}=\mathrm{Cl}, \mathrm{Br}, \mathrm{I})$ Compounds for Photovoltaic and Photocatalytic Applications. Phys. Chem. Chem. Phys. 17 (3), 2199-2209. doi:10.1039/c4cp04666h

Miyake, T., Zhang, P., Cohen, M. L., and Louie, S. G. (2006). Quasiparticle Energy of Semicore D Electrons in ZnS: Combined LDA+U and GW Approach. Phys. Rev. $B 74$ (24), 245213. doi:10.1103/physrevb.74.245213

Mosconi, E., Amat, A., Nazeeruddin, M. K., Grätzel, M., and De Angelis, F. (2013). First-Principles Modeling of Mixed Halide Organometal Perovskites for Photovoltaic Applications. J. Phys. Chem. C 117 (27), 13902-13913. doi:10. $1021 /$ jp 4048659

Ou, M., Tu, W., Yin, S., Xing, W., Wu, S., Wang, H., et al. (2018). Amino-Assisted Anchoring of $\mathrm{CsPbBr} 3$ Perovskite Quantum Dots on Porous G-C3 N4 for Enhanced Photocatalytic CO2 Reduction. Angew. Chem. Int. Ed. 57 (41), 13570-13574. doi:10.1002/anie.201808930

Park, S., Chang, W. J., Lee, C. W., Park, S., Ahn, H-Y., and Nam, K. T. (2016). Photocatalytic Hydrogen Generation from Hydriodic Acid Using Methylammonium lead Iodide in Dynamic Equilibrium with Aqueous Solution. Nat. Energ. 2 (1), 16185. doi:10.1038/nenergy.2016.185

Parr Robert, G., Y. W. (1989). Density-Functional Theory of Atoms and Molecules. New York: Oxford University Press.

Paul, T., Das, D., Das, B. K., Sarkar, S., Maiti, S., and Chattopadhyay, K. K. (2019). $\mathrm{CsPbBrCl} / \mathrm{g}-\mathrm{C} 3 \mathrm{~N} 4$ Type II Heterojunction as Efficient Visible Range Photocatalyst. J. Hazard. Mater. 380, 120855. doi:10.1016/j.jhazmat.2019. 120855

Perdew, J. P., Ernzerhof, M., and Burke, K. (1996). Rationale for Mixing Exact Exchange with Density Functional Approximations. J. Chem. Phys. 105 (22), 9982-9985. doi:10.1063/1.472933

Schneider, J., Jia, H., Muckerman, J. T., and Fujita, E. (2012). Thermodynamics and Kinetics of $\mathrm{CO} 2, \mathrm{CO}$, and $\mathrm{H}+$ Binding to the Metal centre of CO2reductioncatalysts. Chem. Soc. Rev. 41 (6), 2036-2051. doi:10.1039/ clcs15278e

Sehgal, M. L. (2017). Calculation of Spin Orbit Coupling of Tungsten (III) Complexes: A DFT Application. Orient. J. Chem. 33, 1045-1046. doi:10. $13005 / \mathrm{ojc} / 330263$
Shi, Z., and Jayatissa, A. (2018). Perovskites-Based Solar Cells: A Review of Recent Progress, Materials and Processing Methods. Materials 11 (5), 729. doi:10.3390/ ma11050729

Sun, S., Tominaka, S., Lee, J-H., Xie, F., Bristowe, P. D., and Cheetham, Ak. (2016). Synthesis, crystal Structure, and Properties of a Perovskite-Related Bismuth Phase, (NH4)3Bi219. APL Mater. 4 (3), 031101. doi:10.1063/1.4943680

Sutton, R. J., Eperon, G. E., Miranda, L., Parrott, E. S., Kamino, B. A., Patel, J. B., et al. (2016). Bandgap-Tunable Cesium Lead Halide Perovskites with High Thermal Stability for Efficient Solar Cells. Adv. Energ. Mater. 6 (8), 1502458. doi:10.1002/aenm.201502458

Tang, C., Chen, C., Xu, W., and Xu, L. (2019). Design of Doped Cesium lead Halide Perovskite as a Photo-Catalytic CO2 Reduction Catalyst. J. Mater. Chem. A. 7 (12), 6911-6919. doi:10.1039/c9ta00550a

Umari, P., Mosconi, E., and De Angelis, F. (2014). Relativistic GW Calculations on $\mathrm{CH} 3 \mathrm{NH} 3 \mathrm{~Pb} 33$ and $\mathrm{CH} 3 \mathrm{NH} 3 \mathrm{SnI} 3$ Perovskites for Solar Cell Applications. Scientific Rep. 4 (1), 4467. doi:10.1038/srep04467

van Schilfgaarde, M., Kotani, T., and Faleev, S. (2006). Quasiparticle SelfConsistent GW Theory. Phys. Rev. Lett. 96 (22), 226402. doi:10.1103/ PhysRevLett.96.226402

Volonakis, G., Filip, M. R., Haghighirad, A. A., Sakai, N., Wenger, B., Snaith, H. J., et al. (2016). Lead-free Halide Double Perovskites via Heterovalent Substitution of Noble Metals. J. Phys. Chem. Lett. 7 (7), 1254-1259. doi:10.1021/acs.jpclett. $6 \mathrm{~b} 00376$

Volonakis, G., Haghighirad, A. A., Milot, R. L., Sio, W. H., Filip, M. R., Wenger, B., et al. (2017). Cs2InAgCl6: A New Lead-Free Halide Double Perovskite with Direct Band Gap. J. Phys. Chem. Lett. 8 (4), 772-778. doi:10.1021/acs.jpclett. $6 \mathrm{~b} 02682$

Wang, K., Jin, Z., Liang, L., Bian, H., Wang, H., Feng, J., et al. (2019). Chlorine Doping for Black $\gamma$-CsPbI3 Solar Cells with Stabilized Efficiency beyond $16 \%$. Nano Energy 58, 175-182.

Wang, X.-D., Huang, Y.-H., Liao, J.-F., Jiang, Y., Zhou, L., Zhang, X.-Y., et al. (2019). In Situ Construction of a Cs2SnI6 Perovskite Nanocrystal/SnS2 Nanosheet Heterojunction with Boosted Interfacial Charge Transfer. J. Am. Chem. Soc. 141 (34), 13434-13441. doi:10.1021/jacs.9b04482

Wang, X., He, J., Li, J., Lu, G., Dong, F., Majima, T., et al. (2020). Immobilizing Perovskite $\mathrm{CsPbBr} 3$ Nanocrystals on Black Phosphorus Nanosheets for Boosting Charge Separation and Photocatalytic CO2 Reduction. Appl. Catal. B: Environ. 277, 119230. doi:10.1016/j.apcatb.2020.119230

Wu, Y., Wang, P., Zhu, X., Zhang, Q., Wang, Z., Liu, Y., et al. (2018). Composite of $\mathrm{CH} 3 \mathrm{NH} 3 \mathrm{PbI} 3$ with Reduced Graphene Oxide as a Highly Efficient and Stable Visible-Light Photocatalyst for Hydrogen Evolution in Aqueous HI Solution. Adv. Mater. 30 (7), 1704342. doi:10.1002/adma.201704342

Xiaobo, C., Shen, S., Guo, L., and Mao, S. S. (2010). Semiconductor-based Photocatalytic Hydrogen Generation. Chem. Rev. 110 (11), 6503-6570. doi:10.1021/cr1001645

Xu, Y.-F., Yang, M.-Z., Chen, B.-X., Wang, X.-D., Chen, H.-Y., Kuang, D.-B., et al. (2017). A CsPbBr3 Perovskite Quantum Dot/Graphene Oxide Composite for Photocatalytic CO2 Reduction. J. Am. Chem. Soc. 139 (16), 5660-5663. doi:10. 1021/jacs.7b00489

Yan, J., Qiu, W., Wu, G., Heremans, P., and Chen, H. (2018). Recent Progress in 2D/quasi-2D Layered Metal Halide Perovskites for Solar Cells. J. Mater. Chem. A. 6 (24), 11063-11077. doi:10.1039/c8ta02288g

Ye, S., Wang, R., Wu, M.-Z., and Yuan, Y.-P. (2015). A Review on G-C3n4 for Photocatalytic Water Splitting and CO2 Reduction. Appl. Surf. Sci. 358, 15-27. doi:10.1016/j.apsusc.2015.08.173

Yu, C.-J. (2019). Advances in Modelling and Simulation of Halide Perovskites for Solar Cell Applications. J. Phys. Energ. 1 (2). doi:10.1088/2515-7655/aaf143

Yu, Z. B., Xie, Y. P., Liu, G., Lu, G. Q., Ma, X. L., and Cheng, H.-M. (2013). Selfassembled $\mathrm{CdS} / \mathrm{Au} / \mathrm{ZnO}$ Heterostructure Induced by Surface Polar Charges for Efficient Photocatalytic Hydrogen Evolution. J. Mater. Chem. A. 1 (8), 2773-2776. doi:10.1039/c3ta01476b

Yu, Z., Chen, X.-Q., Kang, X., Xie, Y., Zhu, H., Wang, S., et al. (2018). Noninvasively Modifying Band Structures of Wide-Bandgap Metal Oxides to Boost Photocatalytic Activity. Adv. Mater. 30 (14), 1706259. doi:10.1002/ adma.201706259

Yue, D., Zhang, T., Wang, T., Yan, X., Guo, C., Qian, X., et al. (2020). Potassium Stabilization of Methylammonium lead Bromide Perovskite for Robust Photocatalytic H2 Generation. EcoMat 2 (1). doi:10.1002/eom2.12015 
Zeng, S., Kar, P., Thakur, U. K., and Shankar, K. (2018). A Review on Photocatalytic CO2 Reduction Using Perovskite Oxide Nanomaterials. Nanotechnology 29 (5), 052001. doi:10.1088/1361-6528/aa9fb1

Zhang, J., Bai, D., Jin, Z., Bian, H., Wang, K., Sun, J., et al. (2018). 3D-2D-0D Interface Profiling for Record Efficiency All-Inorganic CsPbBrI2 Perovskite Solar Cells with Superior Stability. Adv. Energ. Mater. 8 (15), 1703246. doi:10. 1002/aenm.201703246

Zhang, Q., Ting, H., Wei, S., Huang, D., Wu, C., Sun, W., et al. (2018). Recent Progress in lead-free Perovskite (-like) Solar Cells. Mater. Today Energ. 8, 157-165. doi:10.1016/j.mtener.2018.03.001

Zhao, Y., Wang, Y., Liang, X., Shi, H., Wang, C., Fan, J., et al. (2019). Enhanced Photocatalytic Activity of Ag-CsPbBr3/CN Composite for Broad Spectrum Photocatalytic Degradation of Cephalosporin Antibiotics 7-ACA. Appl. Catal. B: Environ. 247, 57-69. doi:10.1016/j.apcatb.2019.01.090

Zhao, Z., Wu, J., Zheng, Y.-Z., Li, N., Li, X., Ye, Z., et al. (2019). Stable Hybrid Perovskite $\mathrm{MAPb}(\mathrm{I} 1-\mathrm{Br}) 3$ for Photocatalytic Hydrogen Evolution. Appl. Catal. B: Environ. 253, 41-48. doi:10.1016/j.apcatb.2019.04.050
Zhu, X.-Y., and Podzorov, V. (2015). Charge Carriers in Hybrid Organic-Inorganic Lead Halide Perovskites Might Be Protected as Large Polarons. J. Phys. Chem. Lett. 6 (23), 4758-4761. doi:10.1021/acs.jpclett.5b02462

Zhu, X., Lin, Y., Martin, J. S., Sun, Y., Zhu, D., and Yan, Y. (2019). Lead Halide Perovskites for Photocatalytic Organic Synthesis. Nat. Commun. 10 (1), 2843. doi:10.1038/s41467-019-10634-x

Conflict of Interest: The authors declare that the research was conducted in the absence of any commercial or financial relationships that could be construed as a potential conflict of interest.

Copyright (c) 2021 Liu, Fan and Huang. This is an open-access article distributed under the terms of the Creative Commons Attribution License (CC BY). The use, distribution or reproduction in other forums is permitted, provided the original author(s) and the copyright owner(s) are credited and that the original publication in this journal is cited, in accordance with accepted academic practice. No use, distribution or reproduction is permitted which does not comply with these terms. 